\title{
The differentiation effect of bone morphogenetic protein (BMP) on human amniotic epithelial stem cells to express ectodermal lineage markers
}

\author{
Felor Biniazan ${ }^{1,2} \cdot$ Asma Manzari-Tavakoli $^{3} \cdot$ Fahimeh Safaeinejad $^{1} \cdot$ Ali Moghimi $^{3} \cdot$ Farzad Rajaei $^{2} \cdot$ Hassan Niknejad $^{1}$ \\ Received: 10 July 2019 / Accepted: 12 August 2020 \\ (C) Springer-Verlag GmbH Germany, part of Springer Nature 2020
}

\begin{abstract}
Stem cells are a promising tool for treatment of a variety of degenerative diseases. Human amniotic epithelial stem cells (hAECs) have desirable and unique characteristics that make them a proper candidate for cell therapy. In this study, we have investigated the effects of BMP-4 (bone morphogenetic protein-4) and its inhibition on differentiation of AECs into ectodermal lineages. Analysis of AEC-derived ectodermal lineages (neurons and keratinocytes) was performed by using flow cytometry technique for Map2 and $\beta$-tubulin (as neuron markers), Olig2 and MBP (as oligodendrocyte markers), and K14 and K10 (as keratinocyte markers). The results of this study illustrated that noggin (as BMP antagonist), BMP4, and both BMP4 and heparin (together or separately) increased neural and keratinocyte marker expression, respectively. The expression of markers MAP2, olig2, and K14 in hAECs has been significantly decreased 21 days after exposure to differentiation medium (without growth factors) compared with isolation day, which supports the hypothesis that AECs can be dedifferentiated into pluripotent cells. Moreover, activation and inhibition of BMP signaling have no effects on viability of hAECs. The results of this study showed that BMP signaling and its inhibition are the key factors for ectodermal lineage differentiation of amnion-derived stem cells.
\end{abstract}

Keywords BMP4 $\cdot$ Amniotic epithelial cell $\cdot$ Differentiation $\cdot$ Keratinocyte $\cdot$ Neuronal lineage $\cdot$ Glia

\section{Introduction}

Recently, cell therapy holds a great promise for the treatment of various diseases such as neurodegenerative diseases (e.g., spi-

Felor Biniazan, Asma Manzari-Tavakoli, and Fahimeh Safaeinejad are alphabetically ordered co-first authors

Ali Moghimi

moghimi@um.ac.ir

Farzad Rajaei

farzadraj@yahoo.co.uk

$\triangle$ Hassan Niknejad

niknejad@sbmu.ac.ir

1 Department of Pharmacology, School of Medicine, Shahid Beheshti University of Medical Sciences, Tehran, Iran

2 Cellular and Molecular Research Center, Qazvin University of Medical Science, Qazvin, Iran

3 Department of Biology, Faculty of Science, Ferdowsi University of Mashhad, Mashhad, Iran nal cord injury, multiple sclerosis, and Parkinson disease) and wound healing. Using proper cell source for this purpose is very important. Human amniotic epithelial cells (hAECs), which are isolated from human placenta, have favorable and unique characteristics that make them highlighted and attractive for cell therapy. Remarkable properties of hAECs are low immunogenicity, non-tumorigenicity, secretion of several growth factors such as epithelial growth factor (EGF), keratinocyte growth factor (KGF), transforming growth factors (TGF $\beta$ ) (Niknejad et al. 2008; Si et al. 2015; Zhang et al. 2017), as well as immunomodulatory and anti-inflammatory features. Moreover, these cells can be achieved in extensive amounts without ethical concerns in comparison with the other fetal stem cells (Liu et al. 2012). Furthermore, AECs express stem cell surface markers and the pluripotency transcription factors such as octamerbinding protein 4 (Oct-4), Nanog, Lefty A, sex determining region Y-box 2 (SOX-2), and teratocarcinoma-derived growth factor 1 (TDGF1) (Maymó et al. 2018). hAECs are able to differentiate to all three germ layers of endodermal (pancreatic and hepatic cells), mesodermal (cardiac cells), and ectodermal (nervous system and skin) lineages (Wang et al. 2018). 